\title{
Sicherheit für die Zukunft - Pathogeninaktivierung von Thrombozytenkonzentraten
}

\section{Symposium im Rahmen des 36. Jahreskongresses der Deutschen Gesellschaft für Transfusionsmedizin und Immunhämatologie e.V.}

\author{
Innsbruck, 18. September 2003
}

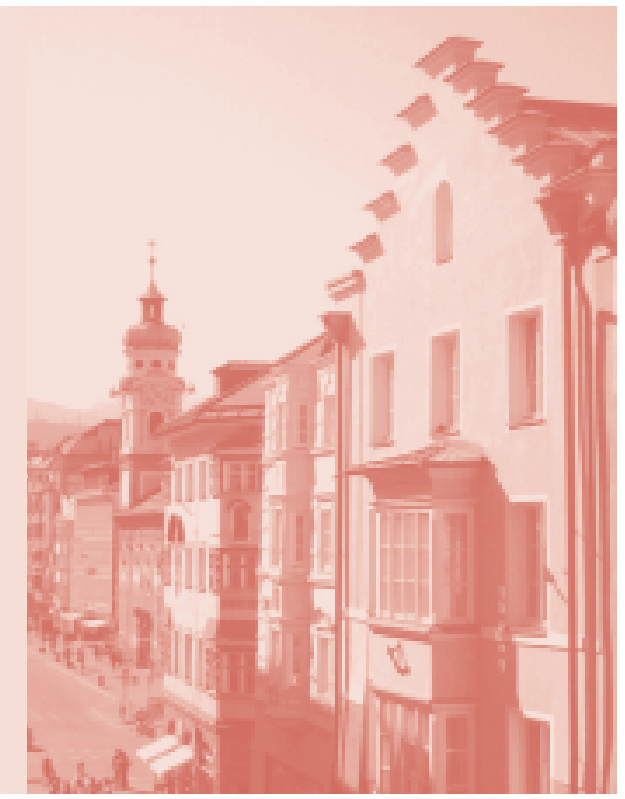

Zwar ist die Sicherheit bei der Übertragung von Blutprodukten höher als je zuvor, doch ein Restrisiko, Infektionen zu übertragen, bleibt bestehen. Zudem muss mit weiteren unbekannten Erregern gerechnet werden, wie die aktuellen Erfahrungen mit West-Nil- (WNV) oder Coronaviren gezeigt haben. Daher sind immer neue Testverfahren zur spezifischen Identifikation einzelner Erreger nötig. Darüber hinaus sind innovative Ansätze notwendig, um neue Krankheitserreger schnell und sicher zu eliminieren. Ein viel versprechender Weg zur Erhöhung der Blutsicherheit wurde jüngst mit der Entwicklung des INTERCEPT Blood Systems für Thrombozyten eingeschlagen, das in den ersten Blutbanken in Europa bereits etabliert ist.

Dr. David A. Leiby, Washington DC, USA, erinnerte daran, dass ständig neue Pathogene entdeckt werden: Anfang der 1980er Jahre war es das «Human Immunodeficiency Virus» (HIV), das die Immunschwäche AIDS (Acquired Immune Deficiency Syndrome) verursacht, in den 1990er Jahren Prionen als Auslöser der neuen Variante der Creutzfeldt-Jakob-Krankheit sowie WNV und jüngst Coronaviren, die zum Severe Acute Respiratory Syndrome (SARS) führen. «Wir können innerhalb von $24 \mathrm{~h}$ per Flugzeug fast überall auf der Erde sein, folglich kann auch jede Erkrankung in der gleichen Zeit zu uns gelangen», meinte Leiby und erinnerte an das plötzliche Auftreten des Ebolavirus. Zu einem veränderten Risikoprofil, das die Verbreitung von Erregern begünstigt, tragen auch die Adaption und Veränderungen der Krankheitserreger selbst und eine veränderte Spender-Demographie bei (höheres Reiseaufkommen und Fernreisen in Endemiegebiete, Einschleppen von Pathogenen durch infizierte Reisende und Immigranten). Leiby griff drei Beispiele - Plasmodien, Babesien und WNV - auf, die seiner Meinung nach auch für die Blutsicherheit in Europa von Bedeutung sind bzw. werden könnten, und erläuterte anhand dieser Beispiele, warum neue Verfahren unerlässlich sind.

\section{Malaria-Gefahr durch asymptomatische Spender}

Obwohl Europa kein Endemiegebiet für Malaria ist, finden sich auch hier Erkrankungsfälle, darunter die «Flughafenmalaria», wie Leiby sie nannte. Im Jahr 2002 belief sich die Zahl der gemeldeten Malariafälle in Europa auf rund 12 000, davon entfielen laut Leiby 861 Erkrankungen auf Deutschland. Da die Malariaerreger Plasmodium falciparum, Plasmodium vivax, Plasmodium malariae und Plasmodium ovale, die durch die Anophelesmücke übertragen werden, nach Infektion in menschlichen Erythrozyten und Leberzellen zu finden sind, muss von einer potentiellen Übertragung durch asymptomatische Blutspender ausgegangen werden.

Leiby machte darauf aufmerksam, dass Erkrankungen wie Malaria aufgrund veränderter Umweltbedingungen und Klimaveränderungen auch in Nichtendemiegebieten Einzug halten können. Denn der ursprünglich in den Tropen beheimatete Überträger der Malaria, die Anophelesmücke, ist heute auch in anderen Lebensräumen, z.B. in den USA, anzutreffen.

\section{West-Nil-Viren im Vormarsch}

Die Übertragung von WNV erfolgt durch Stechmücken. Die natürlichen Wirte dieses Flavivirus sind Vögel, darunter auch Zugvögel. Allerdings können auch Wiederkäuer, Pferde und Menschen klinisch inapparente Infektionen durchlaufen. Beim Menschen kann eine Infektion entweder symptomlos sein oder zu erkältungsähnlichen Symptomen bzw. zur WNV-Enzephalitis und zum Tod führen. Eine spezifische medikamentöse Therapie gibt es nicht. So kam es Ende der 1980er Jahre im Nildelta zu einer Epidemie, bei der zahlreiche Menschen schwer erkrankten. Im Jahre 1999 trat das Virus dann erstmals an der nordamerikanischen Ostküste auf (vermutlich über Vogeltrans-

\section{S. Karger}

Medical and Scientific

Publishers

Basel $\cdot$ Freiburg

Paris $\cdot$ London

New York · Bangalore

Bangkok $\cdot$ Singapore

Tokyo $\cdot$ Sydney

\section{Beilage zu \\ Transfusion Medicine and Hemotherapy

Band 30, Heft 6, November 2003

Band 26, Heft 6, Dezember 2003 
Tab. 1. Transfusionsassoziierte Krankheitserreger

\begin{tabular}{|c|c|c|}
\hline Familie & Erkrankung & $\begin{array}{l}\text { Routine- } \\
\text { Screening }\end{array}$ \\
\hline \multicolumn{3}{|l|}{ Viren } \\
\hline \multicolumn{3}{|l|}{ Hepatitisviren } \\
\hline HBV & Hepatitis & + \\
\hline $\mathrm{HCV}$ & Hepatitis & + \\
\hline $\mathrm{HEV}$ & Hepatitis & - \\
\hline HGV & Hepatitis & - \\
\hline \multicolumn{3}{|l|}{ Retroviren } \\
\hline HIV-1 und -2 & AIDS & + \\
\hline HTLV-I und -II & $\begin{array}{l}\text { maligne Lymphoproliferation, } \\
\text { Neuropathie }\end{array}$ & $(+)$ \\
\hline \multicolumn{3}{|l|}{ Herpesviren } \\
\hline CMV & $\begin{array}{l}\text { CMV-Retinitis, Hepatitis, } \\
\text { Pneumonie }\end{array}$ & $(+)$ \\
\hline EBV & Epstein-Barr-Syndrom & $(+)-$ \\
\hline HHV-8 & Kaposi-Sarkom & \\
\hline \multicolumn{3}{|l|}{ Parvovirus } \\
\hline B19 & aplastische Anämie & - \\
\hline
\end{tabular}

Bakterien

Gramnegative Sepsis

Grampositive Sepsis

Treponema pallidum Syphilis

Borrelia burgdorferi

Borreliose (Lyme)

Rocky-Mountain-Fieber

Rickettsia rickettsi

Rocky-Mountain-Fieber

\section{Parasiten}

Trypanosoma cruzi

Babesia microti

Leishmania donovani

Plasmodium spp.

\section{Chagas-Erkrankung}

Babesiose

Leishmaniose

Malaria

HBV = Hepatitis-B-Virus; HCV = Hepatitis-C-Virus; HEV = Hepatitis-E-Virus; HGV = Hepatitis-G-Virus; HHV-8 = humanes Herpesvirus 8; HTLV-I und -II = humane T-Zell-Leukämie-Viren Typ I and II.

porte eingeschleppt) und hat sich seither rasch über fast alle Bundesstaaten bis in den Westen der USA ausgebreitet. In den USA gingen 2002 allein 23 WNV-Infektionen beim Menschen auf das Konto von Bluttransfusionen, warnte Leiby. Die Mortalitätsrate war hoch: 6 Patienten starben infolge von Meningoenzephalitiden.

\section{Babesiose}

Babesien (Babesia divergens, Babesia microti) werden durch Zeckenbisse übertragen. Sie befallen die roten Blutkörperchen, rufen Erkältungs- bzw. Malaria-ähnliche Symptome hervor und können bei Älteren, bei Immunsupprimierten und bei Menschen ohne Milz zum Tod führen. Durch Transfusionen kam es seit 1979 weltweit zu über 50 Fällen von Babesiose. Dennoch gibt es zum Nachweis von Babesien keinen zugelassenen Test. Zur Babesienproblematik meinte Leiby: «Wir wissen, dass wir
Tab. 2. Inaktivierungsverfahren für pathogene Erreger in Blutprodukten

\begin{tabular}{|c|c|c|}
\hline & \multicolumn{2}{|c|}{ Klinischer Status } \\
\hline & Thrombozyten & Plasma \\
\hline \multicolumn{3}{|c|}{ Photodynamische Verfahren } \\
\hline Riboflavin + UV & Phase I & präklinisch \\
\hline Phenothiazin & präklinisch $^{\mathrm{a}}$ & eingeführt $\mathrm{t}^{\mathrm{b}}$ \\
\hline \multicolumn{3}{|c|}{ Photochemische Verfahren } \\
\hline $\mathrm{S}-59+\mathrm{UVA}^{\mathrm{c}}$ & $\begin{array}{l}\text { Phase-III-CE- } \\
\text { Zertifikation }\end{array}$ & Phase III \\
\hline \multicolumn{3}{|l|}{ Andere Verfahren } \\
\hline Inactine $\mathrm{T}^{\mathrm{TM}}$ & präklinisch & präklinisch \\
\hline Solvent-Detergent & - & eingeführt \\
\hline \multicolumn{2}{|c|}{ b1,9-Dimethylenblau + sichtbares Licht. } & \\
\hline
\end{tabular}

die Parasiten mit Transfusionen übertragen, aber wir haben keine Testverfahren, um sie zu erkennen.»

\section{Krankheitsübertragung duch Blutprodukte trotz routinemäßig eingesetzter Screening-Verfahren}

Neue Krankheiten werden auch in Zukunft eine Gefahr für die Blutsicherheit darstellen, so Leiby. Eine Gefährdung für Transfundierte besteht grundsätzlich immer dann, wenn unbekannte pathogene Keime auftreten, die durch routinemäßig eingesetzte Screening-Verfahren nicht erfasst werden. Nur wenn die Erreger identifiziert worden sind, können Nachweisverfahren entwickelt werden. Die Entwicklung neuer Testverfahren kostet aber Zeit und geschieht nur dann, wenn feststeht, dass die Erreger auch tatsächlich über Bluttransfusionen übertragen werden können. Die Gefahr ist jedoch erst gebannt, wenn diese neu entwickelten Verfahren auch als routinemäßige Screening-Verfahren implementiert worden sind - allerdings immer nur für den jeweiligen pathogenen Keim. Daher sind dringend neue Ansätze nötig, um Übertragungen durch Blutprodukte zu vermeiden.

\section{Kein Screening auf alle transfusionsassoziierten pathogenen Erreger}

Dr. Susanne Picker, Köln, ergänzte, dass außerdem nicht für alle transfusionsassoziierten pathogenen Erreger routinemäßig Screening-Verfahren durchgeführt werden (Tab. 1). Auch kontaminierte Leukozyten sind ein Problem, da sie die Übertragung zellassoziierter Infektionserreger wie Cytomegalovirus (CMV) und Epstein-Barr-Virus (EBV) ermöglichen. Sie können ebenfalls nichthämolytische febrile Transfusionsreaktionen und transfusionsassoziierte Immunmodulationen (Alloimmunisierung gegen Spender-HLA-Antigene, Graft-versus-hostKrankheit) auslösen.

\section{Diagnostisches Fenster und Spenderbefragung}

Ein Restrisiko besteht zudem aufgrund des diagnostischen Fensters, selbst wenn durch NAT/PCR diese Periode deutlich verkürzt wird. Bei frischen Infektionen greifen die derzeitigen 
Screening-Verfahren erst, wenn genügend Erreger im Blut vorhanden bzw. genügend Antikörper gebildet worden sind. Kurz nach einer Infektion des Spenders liefern sie falsch-negative Ergebnisse.

Ein weiteres Risiko liegt bei den Spendern selbst, denn ein geringer Prozentsatz verschweigt bestimmte Risikofaktoren.

\section{Bakterielle Kontamination}

Ein Problem stellt auch die (bakterielle) Kontamination der Blutproben selbst dar, die in einer amerikanischen Studie [Blajchman: Semin Hematol 2001;38:20-26] bei 1 von 2000 Thrombozytenkonzentraten liegt. Bei 1 von 6 Empfängern kontaminierter Thrombozytenkonzentrate kommt es zu einer Sepsis, wobei jeder Vierte der Betroffenen stirbt.

\section{Innovative Verfahren zur Erregerinaktivierung}

Picker wandte sich daher neuen Verfahren zur Inaktivierung pathogener Keime zu. So können zelluläre Blutprodukte photoinaktivierenden (photodynamischen oder photochemischen) Verfahren unterzogen werden (Tab. 2).

Bei photodynamischen Verfahren finden Riboflavin, Phenothiazine (z.B. Thionin oder Methylenblau), Zyanine oder Porphyrine (z.B. Hämatoporphyrin und Benzoporphyrin) Anwendung. Während Methylenblau jedoch laut Picker Faktor VIII schädigt und nur ein mäßig breites Wirkspektrum hat, verfügt 1,9-Dimetymethylenblau über eine stärkere intrazelluläre Wirkung. Porphyrine besitzen ebenfalls nur ein mäßig breites Wirkspektrum. Auch Zyanine haben ein nur eingeschränktes Wirkspektrum und zudem das gleiche Absorptionsmaximum wie Hämoglobin und wirken auf Erythrozyten sowie Thrombozyten phototoxisch. Bei Thionin ist die Mutagenität nicht geklärt, und bei PEN 110 (Inactine ${ }^{T M}$ ) ist laut Picker das toxikologische Profil der Restsubstanz nicht geklärt.

Riboflavin mit seinem breiten Wirkspektrum räumte Picker bei fehlender Gentoxizität und fehlender akuter Toxizität gute Chancen ein. Riboflavin, ergänzte sie, muss zudem nicht entfernt werden. Für diese Substanz liegen allerdings erst Erfahrungen aus Phase-I-Studien vor, während sich andere Verfahren bereits etabliert haben. Vitamin B2 und Psoralene - beides Stoffe bzw. Stoffgruppen, die auch mit der täglichen Nahrung aufgenommen werden - sind laut Picker, die einzigen Substanzen, für die positive Effekte eindeutig belegt sind. Psoralene wurden bisher in photochemischen Verfahren intensiv untersucht und haben der Referentin zufolge ein gutes toxisches Profil und viel versprechende In-vitro- und In-vivo-Daten. Für die Implementierung innovativer photochemischer Verfahren zur Pathogeninaktivierung von Blutprodukten spricht laut Picker:

- das hohe Sicherheitsbedürfnis für Blutprodukte,

- die Inaktivierung hüllenloser Viren (Parvovirus B19),

- die Wirkung gegen bakterielle Kontamination,

- die Wirkung gegen zellassoziierte Viren (CMV) und

- die Reduktion leukozytenabhängiger Immunmodulation.

Noch zu klären sind die Kosteneffektivität und die Langzeitwirkungen der Restsubstanz.

\section{INTERCEPT Blood System für Thrombozyten in Europa bereits eingesetzt}

Das INTERCEPT Blood System für Thrombozyten (Abb. 1) wird in den Blutbanken einiger europäischer Länder wie Norwegen, Belgien, Portugal, Spanien und Italien bereits zur Inak-

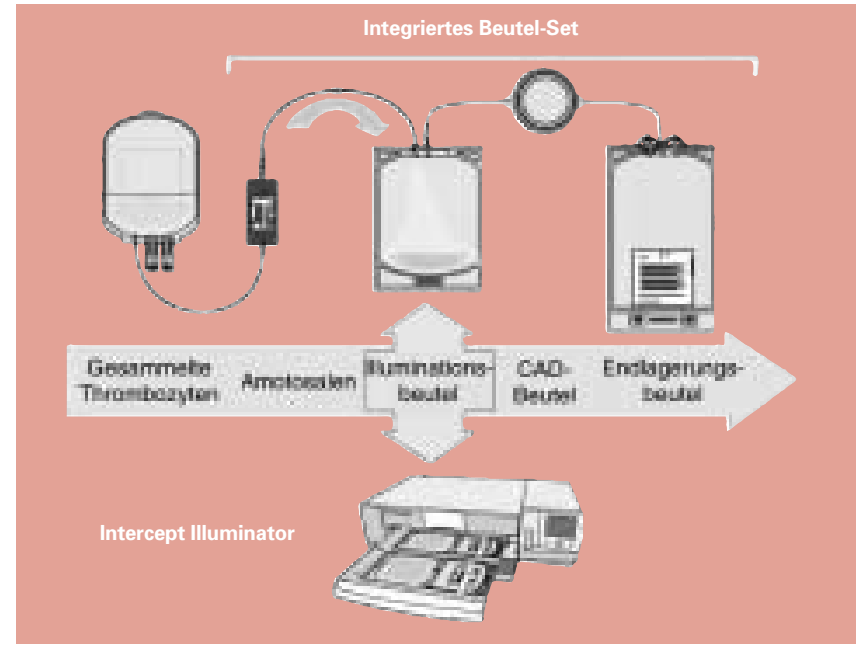

Abb. 1. INTERCEPT Blood System für Thrombozyten.

tivierung pathogener Keime eingesetzt. In Deutschland wurden in 13 Zentren 16 Validierungen durchgeführt. Die Zulassung des Paul-Ehrlich-Instituts steht derzeit noch aus.

\section{Bekannte und unbekannte pathogene Keime werden inaktiviert}

Picker erklärte das Wirkprinzip dieses innovativen Systems, mit dem behüllte und unbehüllte Viren, gramnegative und grampositive Bakterien sowie neue und unbekannte Erreger inaktiviert werden können, ohne dass es einer besonderen Abstimmung auf die Besonderheiten bestimmter Mikroorganismen bedarf. Der Ansatz ist immer derselbe. Für Thrombozyten ist das Verfahren unschädlich. Durch Inaktivierung von Leukozyten wird auch das Risiko unerwünschter Transfusionsreaktionen reduziert.

Das System basiert auf der Helinx ${ }^{\circledR}$-Technologie und der photochemischen Aktivierung von Amotosalen $\mathrm{HCl}$ (S-59), das dem fertigen Thrombozytenkonzentrat zugesetzt wird. S-59 ist ein synthetisch hergestelltes Psoralen mit hoher Affinität zu Nukleinsäuren, mit denen es zunächst eine reversible Verbindung eingeht. Nach UVA-Bestrahlung im Illuminator wird Amotosalen aktiviert und es entstehen irreversible Kreuzvernetzungen, die die Replikation und Erregervermehrung effektiv und nachhaltig unterbinden. Das System ist folglich auch in der Lage, menschliche Coronaviren in Blutprodukten zu inaktivieren.

\section{Sicherheit und Wirksamkeit pathogeninaktivierter Thrombozytenkonzentrate}

Um die Äquivalenz Erreger-inaktivierter Plättchenkonzentrate mit konventionellen Blutprodukten zu demonstrieren, wurden jüngst zwei Studien durchgeführt:

\section{euroSPRITE}

Zur Prüfung von Wirksamkeit und Sicherheit des innovativen INTERCEPT Blood Systems für Thrombozyten wurde in Europa die euroSPRITE-Studie (European S-59 Platelets in Recovery in Thrombocytopenic Patients) mit 103 über 16-jährigen 
Thrombozytopeniepatienten durchgeführt, die entweder einer Therapie mit konventionellen Thrombozytenkonzentraten oder mit solchen, die mit dem INTERCEPT Blood System behandelt worden waren, unterzogen wurden. Die Transfusionen erfolgten als supportive Maßnahmen über einen Zeitraum von 8 Wochen. Der Nachbeobachtungszeitraum umfasste 4 Wochen. Dabei zeigten die mit dem photochemischen Verfahren behandelten Thrombozytenkonzentrate eine ähnliche Wirksamkeit wie herkömmliche Präparate. Blutungsprävention, klinische Hämostase, Sicherheit und Transfusionsreaktionen waren miteinander vergleichbar.

\section{SPRINT}

In den USA wurde die SPRINT-Studie (S-59 Platelet Recovery in Thrombocytopenia) mit 645 Patienten, die Test- bzw. Referenzkonzentrate erhielten, initiiert. Im Gegensatz zur europäischen euroSPRITE-Studie wurden in diese nordamerikanische prospektive, randomisierte, doppelblinde, multizentrische Untersuchung hauptsächlich transplantierte Patienten eingeschlossen, erläuterte Prof. Dr. Richard J. Benjamin, Boston, MA, USA.

Thrombozytentransfusionen erfolgten über einen Zeitraum von 28 Tagen, an den sich eine Beobachtungsphase von 7 Tagen anschloss. Bei Bedarf bekamen die Patienten in einem zweiten 28tägigen Zyklus erneut Transfusionen. Der Nachbeobachtungszeitraum im Anschluss an dieses Intervall betrug ebenfalls 7 Tage. Im ersten Zyklus erhielten 645 Patienten insgesamt 4719 Transfusionen. In einem zweiten Zyklus wurden 91 Patienten behandelt. Dabei dienten die Plättchentransfusionen bei den meisten Patienten prophylaktischen Zwecken, und nur ein geringer Prozentsatz dieser schwer kranken Patienten erhielt sie wegen aktiver Blutungen. Der primäre Endpunkt der Studie war definiert als der Anteil an Patienten mit Blutungen (Grad 2, WHO-Kriterien), die als «mild» bis «mäßig» gelten und bei denen es nicht innerhalb von $24 \mathrm{~h}$ einer Transfusion bedarf. Wenn man Blutungen zweiten Grades behandelt, möchte man insbesondere vehementere Ereignisse vermeiden, und so war einer der sekundären Endpunkte der Studie definiert als Anteil der Patienten mit stärkeren bzw. lebensbedrohlichen Blutungen (Grad 3 und 4). Auch die Anzahl der benötigten Erythrozytentransfusionen, die Anzahl, Lokalisation und Dauer der Blutungen zweiten Grades, die Zeit bis zum Beginn der Blutungen, der Anteil der Patienten mit maximalen Blutungen Grad 2, die Thrombozytenzahl 1 sowie $24 \mathrm{~h}$ nach Transfusion, das Intervall zwischen den Thrombozytentransfusionen, die Anzahl der Thrombozytenkonzentrate und die Dauer der supportiven Maßnahmen wurden dokumentiert.

Die Daten dieser umfangreichen Untersuchung zeigten, dass die Wirksamkeit der mit dem INTERCEPT Blood System für Thrombozyten behandelten Konzentrate den konventionell hergestellten Konzentraten in Hinblick auf die Häufigkeit, die Zeit bis zum Einsetzen sowie die Lokalisationen der Blutungen zweiten Grades ebenbürtig war. Blutungen (Grad 2) traten bei $58,5 \%$ der Patienten in der Testgruppe und bei $57,5 \%$ in der Referenzgruppe auf $(\mathrm{p}=0,80)$ und setzten in beiden Studienarmen auch in etwa zeitgleich ein. Gleiches galt für die Anzahl an Patienten mit maximalen Blutungen zweiten Grades. Auch unerwünschte Ereignisse inklusive Todesfälle waren in beiden Gruppen etwa gleich häufig - es gab keine signifikanten Unterschiede. Erstaunlicherweise kam es sogar in der Referenzgruppe bei mehr Patienten zu Blutungen dritten oder vierten Grades (4,1 versus $6,1 \%)$, die Unterschiede waren jedoch nicht sta- tistisch signifikant. Transfusionsreaktionen waren allerdings in der Referenzgruppe (4,9\%) signifikant häufiger als im Testarm $(3,0 \% ; p=0,02)$. Antikörper gegen (S-59 assoziierte) Neo-Antigene dagegen wurden nach Transfusionsexposition nicht gefunden. In der Testgruppe kam es jedoch zu einer Verkürzung der Transfusionsintervalle (1,9 versus 2,4 Tage); somit waren bei diesen Patienten auch mehr Plättchentransfusionen nötig $(8,4$ versus 6,2 Transfusionen pro Patient). Auch die Dauer der Transfusionen war in der Testgruppe länger (11,8 versus 10,6 Tage). Das könnte damit zu tun haben, dass die Thrombozytendosen ungleichmäßig verteilt waren und dass unter den Testpatienten mehr niedrig dosierte Thrombozytenkonzentrate zu finden waren. Die Dosierungen waren inkonsistent, da ein Vorläufermodell verwendet wurde, auf das der Verlust von Thrombozyten zurückzuführen war. Die Referenzthrombozyten hingegen wiesen konsistentere Dosen auf.

Die mit dem INTERCEPT Blood System behandelten Thrombozyten beugten Blutungen bei Patienten mit schwerer Thrombozytopenie effektiv vor und müssen den Vergleich mit konventionellen Präparaten nicht scheuen. Die Daten der SPRINTStudie bestätigen Benjamin zufolge die Hypothese der statistischen Äquivalenz.

\section{Y.C. Zwick, München}

\section{Referenten}

David A. Leiby, PhD, Washington DC, USA

Dr. Susanne Picker, Köln, Deutschland

Dr. Andy Bailey, Wien, Österreich

Univ. Prof. Dr. Gabriele Stiegler, Wien, Österreich

Richard J. Benjamin, MBChB, PhD, Boston, MA, USA

\section{Impressum}

\section{Beilage zu}

Transfusion Medicine and Hemotherapy 30 | 6 | 03

ONKOLOGIE 26 | 6 | 03

(C) 2003 by S. Karger Verlag, Verlag für Medizin und Naturwissenschaften GmbH, Lörracher Straße 16a, D-79115 Freiburg

Titelfoto: Y.C. Zwick

Mit freundlicher Unterstützung durch Baxter Deutschland GmbH, Unterschleißheim.

Der Verlag und die Herausgeber der Zeitschrift übernehmen keine Verantwortung für diese Rubrik.

Die Wiedergabe von Gebrauchsnamen, Handelsnamen, Warenbezeichnungen usw. in dieser Beilage berechtigt auch ohne besondere Kennzeichnung nicht zur Annahme, dass solche Namen im Sinne der Warenzeichen- und MarkenschutzGesetzgebung als frei zu betrachten wären und daher von jedermann benutzt werden dürfen. Für Angaben von Dosierungsanweisungen und Applikationsformen kann vom Verlag keine Haftung übernommen werden. Derartige Angaben müssen vom jeweiligen Anwender im Einzelfall anhand anderer Literaturstellen auf ihre Richtigkeit überprüft werden.

Alle Rechte vorbehalten.

Ohne schriftliche Genehmigung des Verlags dürfen diese Publikation oder Teile daraus nicht in andere Sprachen übersetzt oder in irgendeiner Form mit mechanischen oder elektronischen Mitteln reproduziert oder auf einem Datenträger oder Computersystem gespeichert werden.

Druck: Konkordia GmbH · Das Medienunternehmen 